\title{
State-of-the-Art in Immobilized Polymer Stationary Phases for High-Performance Liquid Chromatography
}

\author{
Anizio M. Faria, ${ }^{a, b}$ Carol H. Collins ${ }^{b}$ and Isabel C. S. F. Jardim ${ }^{* . b}$ \\ ${ }^{a}$ Química-FACIP, Universidade Federal de Uberlândia, 38302-000 Ituiutaba-MG, Brazil \\ ${ }^{b}$ Instituto de Química, Universidade Estadual de Campinas, CP 6154, 13083-970 Campinas-SP, Brazil
}

\begin{abstract}
O aumento da estabilidade química e a redução da alta atividade de grupos residuais têm sido, nas últimas quatro décadas, os principais focos de pesquisas na preparação de fases estacionárias para cromatografia líquida de alta eficiência na modalidade de fase reversa (CLAE-FR). Novas e eficientes estratégias de modificação da superfície da sílica e a introdução de novos materiais como suportes cromatográficos têm minimizado estas limitações e possibilitado uma crescente popularização da CLAE-FR. Neste trabalho será apresentada uma visão geral do estado da arte de uma das estratégias de preparo de fases estacionárias que vem contornando tais limitações da CLAE-FR; as fases estacionárias baseadas na imobilização de polímeros orgânicos pré-sintetizados sobre partículas de sílica e sílica metalizada (zirconizada e titanizada). Uma consideração especial será dada ao desenvolvimento destas fases estacionárias ocorrido no Brasil nos últimos 15 anos.
\end{abstract}

Increasing stability and reducing the high activity of residual groups from stationary phases have been the main focuses of research on the preparation of reversed-phase high-performance liquid chromatography (RP-HPLC) phases over the last four decades. New and more efficient strategies for modifying the silica surface as well as the introduction of new chromatographic supports have minimized these effects and have enabled the increasing popularization of RP-HPLC. In this paper, an overview will be given of the state-of-the-art in stationary phases for RP-HPLC based on immobilization of pre-synthesized organic polymers on both silica and metalized-silica supports. Special consideration is given to the development of HPLC stationary phases in Brazil in the past 15 years.

Keywords: polymer-coated stationary phases, high-performance liquid chromatography, metalized silica supports

\section{Introduction}

Today, about 40 years after its appearance, highperformance liquid chromatography (HPLC) is one of the most important analytical techniques for qualitative and, in particular, quantitative determinations. The field of application of liquid chromatography is increasingly broad, covering scientific, commercial and industrial interests in the most diverse areas such as pharmaceuticals, medicine, food, synthetic polymers, environment, etc. The continuous development of stationary phases during this period has been the principal factor responsible for the popularization of HPLC, allowing ever better separations for a wide variety of compounds. Despite the growing number of publications involving HPLC applications, development of stationary phases still occupies a prominent place in the literature.

*e-mail: icsfj@iqm.unicamp.br
The stationary phases most used in HPLC consist of organic chains, for example, octadecylsilane (C18) and octylsilane (C8), chemically bonded to chromatographic silica. These reversed stationary phases (RP), due to the inversion of selectivity with introduction of organic groups onto silica support, represent approximately $85 \%$ of all HPLC applications.

\section{Chemically Bonded Stationary Phases}

Historically, the first stationary phases developed for RP-HPLC used gas chromatographic (GC) column technology for their preparation, as GC had emerged about 20 years earlier and the development of stationary phases had already made significant progress. These GC stationary phases consisted essentially of liquids of low to medium molar mass that were sorbed onto the chromatographic support. ${ }^{1}$ The main difficulty in using similar stationary 
phases in RP-HPLC was to maintain the stationary liquid concentration constant on the chromatographic support. This was not a problem in GC, at least within the temperature limits imposed by the liquid's volatility but, in RP-HPLC, the sorbed phase was removed due to solubility in the mobile phase, especially when the mobile phase flow rates were increased. This fact impeded obtaining repeatable analyses or separation of complex mixtures as it was not feasible to use the gradient elution mode. ${ }^{2}$ Elimination of most of these initial problems with RP-HPLC became possible due to the introduction of chemically bonded stationary phases (CBSP), in which alkylsilane reagents with long carbon chains (C8 and C18) are covalently connected to the support surface, increasing the hydrolytic stability of the resulting stationary phase. ${ }^{2}$ The advent of CBSP was the predominant factor for the development of HPLC.

Several methods have been suggested to promote this covalent attachment of the silylant agent to the silica through the silanol groups, $\mathrm{Si}-\mathrm{OH}$, present on the silica surface. Among the most successful methods can be cited direct esterification, ${ }^{3}$ sequential reactions with thionyl chloride and Grignard reagent ${ }^{4}$ and the silanization method. ${ }^{5-7}$ This latter eventually becoming the primary method for modifying the silica surface, being employed by all manufacturers of HPLC stationary phases. ${ }^{8}$ The silanization method employs a variety of silylant agents having one, two or three substitutable groups, usually halides or alkoxy groups, bonded to a silicon that also has a long chain alkyl group (C8, C18, C30, etc.) (Figure1).
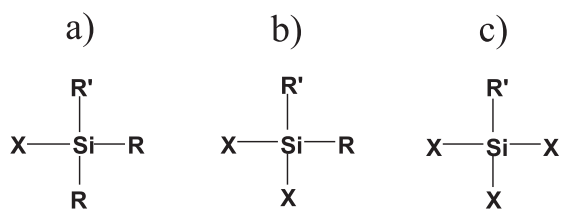

Figure 1. Organosilanes used in the preparation of chemically bonded stationary phases: (a) monofunctional, (b) bifunctional and (c) trifunctional. $\left[\mathrm{R}=\mathrm{CH}_{3} ; \mathrm{R}^{\prime}=\mathrm{C}_{8} \mathrm{H}_{17}, \mathrm{C}_{18} \mathrm{H}_{37}\right.$; etc., $\mathrm{X}=\mathrm{OCH}_{3}, \mathrm{OC}_{2} \mathrm{H}_{5}$ or $\left.\mathrm{Cl}\right]$.

The main advantage offered by the silanization method is the high stability of the attachment between the silylant agent and silica through the siloxane bond (Si-O-Si-C), resulting in stationary phases with higher hydrolytic stability and better column efficiencies due to their rapid mass transfer. ${ }^{2}$

In spite of substantial progress with the CBSP, steric considerations still hamper obtaining dense distributions of alkylsilane molecules on the silica surface, as about $50 \%$ of the silanol groups remain without reacting. ${ }^{89}$ This low bonding density on the silica surface resulted in two historical problems found in RP-HPLC: (i) Applications of silica-based stationary phases are limited to the $2-8 \mathrm{pH}$ range, due to the exposure of part of surface, not modified with the alkylsilane, to attack from constituents in the mobile phases. Below pH 2, the Si-C bonds between silicon and the alkyl group are hydrolyzed, resulting in cleavage of the organic phase attached to the chromatographic support, while, above $\mathrm{pH} \mathrm{8}$, silica dissolution is accelerated, resulting in loss of the support, and (ii) the presence of residual silanol groups that interact strongly with some analytes (silanophilic activity) by an ion exchange mechanism affects the chromatographic retention of these compounds. This fact occurs mainly in the elution of basic compounds, such as a large number of pharmaceutical and bio-organic compounds, due to the high acidity of these silanol groups. ${ }^{9}$

These limitations related to the use of silica-based stationary phases led to two different directions for HPLC stationary phase development: $(i)$ a search for other chromatographic supports as alternatives to silica and (ii) the development of new methods and/or reagents for a more efficient modification of the silica surface. Both of these aimed at obtaining stationary phases with improved chemical stability over a wider $\mathrm{pH}$ range and, therefore, making the separation of a wider range of analytes, including macromolecules and basic compounds, possible. ${ }^{10}$

Several materials were evaluated as possible substitutes for silica, with emphasis on organic polymers ${ }^{11-13}$ and some inorganic oxides such as alumina, ${ }^{14-16}$ titania $^{16-18}$ and zirconia. ${ }^{16,19-22}$ The parameter used by chromatographers for the selection of these materials as chromatographic supports was their chemical stability over a wider $\mathrm{pH}$ range, when compared with silica. However, most stationary phases prepared with these materials do not show the chromatographic performance usually obtained with silicabased phases. In addition, these materials had a number of other drawbacks that do not occur with silica. For example, organic polymer particles have low mechanical strength, restricting their use at higher pressures, and usually show broader peaks due to the slower solute mass transfer in the polymeric stationary phase..$^{23}$ On the other hand, the alternative inorganic oxides present surfaces that are difficult to modify, resulting in even lower densities of organic ligands and more exposure of high reactivity sites, such as the Lewis acid sites on zirconia and titania, that interact with acidic and basic compounds even more strongly than silanol groups. ${ }^{16,24,25}$ Furthermore, these materials have poorly developed preparation technologies and limited commercial availability with respect to their particle forms, sizes and porosities.

More recently, hybrid support particles, containing methyl ${ }^{26,27}$ or ethylene ${ }^{28}$ groups embedded into the silica structure, prepared using the sol-gel process from a mixture 
of tetraethoxysilane (TEOS) and methyltriethoxysilane (MTEOS) or bis(triethoxysilyl)ethane (ETEOS). The presence of these groups in the silica structure confers greater chemical stability, nominally to $\mathrm{pH} 12$, as compared to the bare silica support. In addition, hybrid silica-based stationary phases have less residual activity due to the incorporation of methyl (or ethylene) groups in substitution of some of the residual silanols. On the other hand, the decrease of silanols groups on the silica surface also reduces the attachment points of the silylant agents, resulting in less dense organic substituents while some residual silanols still remains, due to steric hindrance.

Another direction of research in stationary phase preparation has been the development of procedures and reagents for more efficient modification of the silica surface by blocking access to the residual silanols. The use of a second silanization step, substituting the long alkyl chain with a methyl group using reagents such as trimethylchlorosilane (TCMS) and/or hexamethyldisilazane (HMDS), produces the classic "end-capping" reaction" 29,30 and significantly reduces the number of residual silanol groups and the resulting ion-exchange reactions with basic compounds as well as enhancing the stability of the resulting phase. These end-capping reactions continue to be useful for reducing silanol activity even after other preparative procedures, such as those described below, were developed.

Another alternative was the preparation of vertically polymerized stationary phases ${ }^{31,32}$ (Figure 2a) that result from the chemical bond between bifunctional (or trifunctional) silylant agents (Figure 1) and the silanol groups in the presence of traces of water. The presence of water hydrolyses more of the halide or alkoxyl groups, resulting in some interchain bonds. These phases have, in some situations, higher chemical stabilities in acidic and basic conditions than monomeric phases, prepared from a monofunctional alkylsilane (Figure 1a). However, problems with the repeatability of the quantities of alkylsilane chemically attached to the silica due to uncontrolled secondary reactions with trace water results in some lack of repeatability in the retention and selectivity parameters. ${ }^{8}$

To improve the repeatability of chromatographic parameters in polymerized phases, horizontally polymerized stationary phases were developed ${ }^{33,34}$ from the self-assembly of monolayers of the trifunctional alkylsilanes of long $(\mathrm{C} 8, \mathrm{C} 18)$ and short $(\mathrm{C} 1)$ chains (Figure 2b) chemically attached to the silica surface. The literature ${ }^{34}$ suggests that the process of obtaining these stationary phases is highly reproducible, especially when compared to vertically polymerized phases. However, there is no published data showing the repeatability of this preparation process.
Despite a marked improvement in the chemical stability reached by chemically bonded polymeric phases, the major progress in the development of more stable RP-HPLC stationary phases has been made with sterically protected, ${ }^{35}$ bidentate ${ }^{36,37}$ and polar embedded stationary phases. ${ }^{38-41}$

Sterically protected stationary phases were prepared by reaction of a monochloro silylant agent containing a long alkyl group (C8 or C18) with two smaller but bulky alkyl groups (isopropyl or tert-butyl) ${ }^{35}$ substituting the usual methyl groups (Figure 1a, R = isopropyl or tert-butyl). These bulkier groups restrict the access of basic analytes to the residual silanol groups (Figure 2c). However, despite restricting access to the adjacent residual silanols and to the $\mathrm{Si}-\mathrm{C}$ bond itself, these phases have lower ligand densities when compared to conventional CBSP, and are thus recommended for use at low $\mathrm{pH}$ where the silanol groups are not ionized. ${ }^{42}$

Bidentate phases are usually prepared from the chemical attachment to two adjacent silanol groups of silylant agents containing two $\mathrm{C} 18$ groups linked to two silicon atoms separated by a propyl group ${ }^{36,37}$ (Figure 2d). The bidentate reagent produces restricted access of analytes to residual silanols and provides higher chemical stability to this stationary phase, which can be used in mobile phases at $\mathrm{pH} 11 .{ }^{36}$ However, similar to the sterically protected phases, bidentate phases have lower ligand densities on the silica surface and still require a subsequent end-capping reaction to block the residual groups.

Stationary phases with embedded polar groups are based on the chemical attachment to the silanol groups from the silica surface of a silylant agent containing an embedded polar group (amide, ${ }^{38}$ carbamate, ${ }^{39}$ urea, ${ }^{40}$ tiocarbamate ${ }^{41}$ ) inserted after the third methylene group on the alkyl chain (Figure 2e). The main characteristic of this stationary phase is the reduction of silanophilic activity due to the competition between polar groups and residual silanols for the basic components of the samples. ${ }^{8}$ Another mechanism also accepted in the literature is the interaction of polar groups with water molecules from mobile phase, giving an "empirical wall" of water molecules near the silica surface. This layer limits access of basic analytes to the silanol groups. ${ }^{43}$ Embedded polar group stationary phases have good stability and repeatability when using highly aqueous mobile phases. ${ }^{44}$ However, the repeatability of retention parameters for polar, acidic and basic compounds is usually lower than those obtained with conventional CBSP.

Although some of these procedures have offered better protection of the chromatographic support, resulting in improved chemical stability, principally with alkaline mobile phases, stationary phases prepared with these new reagents did not completely solve the problem of residual 

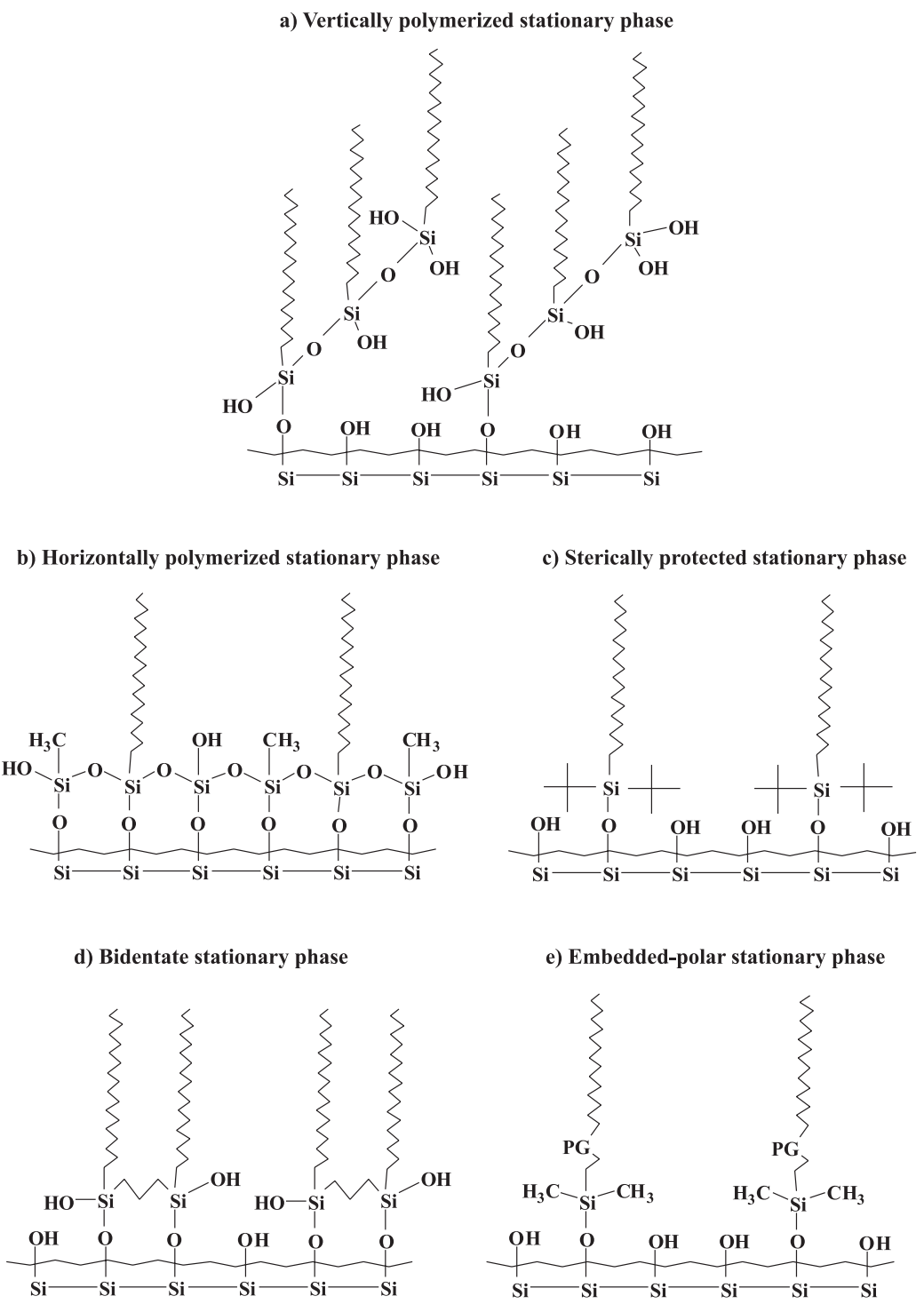

Figure 2. Chemically bonded stationary phases developed to reduce silanophilic activity.

silanols. In most cases, the alkysilane densities on the silica surface were lower than that observed with the classic stationary phase, due to the steric hindrance caused by the higher volume of the new reagent molecules, usually requiring a subsequent "end-capping reaction".

\section{Silica-Based Immobilized Polymer Stationary Phases}

In addition to development of procedures based on the chemical attachment of silylant agents to the silica surface, an alternative is the preparation of stationary phases by the immobilization of pre-synthesized organic polymers on the silica surface..$^{45-47}$ Immobilized polymer stationary phases have several advantages that make them very attractive for use in RP-HPLC, such as better protection of residual silanols (silica) or Lewis acid sites (zirconia, titania), compared to CBSP, minimizing ion exchange interactions with some sample components; more effective protection of the support matrix against the chemical attack of alkaline mobile phases; ease of preparation, etc. ${ }^{16,48}$ Furthermore, the selectivity of these phases depends fundamentally on the functional groups present in the immobilized polymer. Due to the availability of a wide range of polymers with different functionalities, these stationary phases allow a finer adjustment of chromatographic selectivity within a wide range, depending only on a choice of the suitable polymer for the specific separation.

Immobilized polymer stationary phases are normally prepared in two ways: $(i)$ by sorption/immobilization of presynthesized polymer layers ${ }^{45-47,49,50}$ sorbed onto the support surface and in the pores or (ii) by in situ polymerization 
of monomers ${ }^{51,52}$ sorbed onto the support. However, the first procedure has been better studied because of the repeatability of the sorption of the pre-formed polymer onto the support, resulting in better repeatability of the resulting retention parameters from column-to-column.

Basically, the sorbed polymer can form a thin film on the silica surface (Figure 3a) or it can fill the full pore volume (Figure $3 b$ ). It is also possible to have a combination of these two forms. ${ }^{53}$ In both cases, polymer chains can cover the pore walls (by adsorption) without cross-linking between the chains of the polymer (left side of Figures $3 \mathrm{a}$ and $3 \mathrm{~b}$ ) or, under appropriate conditions, the polymer chains can also form a thick, cross-linked layer without chemical attachment to the pore surfaces (center of Figures $3 \mathrm{a}$ and $3 \mathrm{~b}$ ). They can also chemically attach to the pore walls (right side of Figures $3 a$ and $3 b$ ), in addition to cross-linking.

Given these possibilities, the Research Laboratory for Liquid Chromatography of the Institute of Chemistry of Unicamp (LabCrom) initiated, some 20 years ago, studies on the preparation of silica-based immobilized polymer stationary phases. Since this start, stationary phases prepared at LabCrom have been accompanying the developments in RP-HPLC, becoming increasingly competitive.

Initial studies involving the preparation of stationary phases were performed by Anazawa and Jardim. ${ }^{54}$ These authors prepared stationary phases based on sorption of poly(methyloctylsiloxane) (PMOS) on irregular Davisil silica particles $(10 \mu \mathrm{m})$, using the method of static evaporation of solvent. In this method, the polymer is dissolved in an organic solvent, in which the polymer is completely soluble and then appropriate quantities of silica are added to this polymer solution with gentle stirring.

(a)

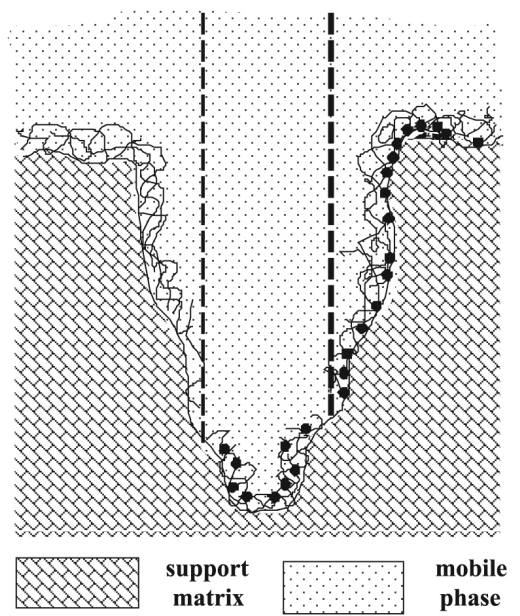

The sorbed material is then placed in a fume hood without stirring until the complete evaporation of organic solvent at room temperature. ${ }^{54,55}$ These new stationary phases were then tested using a mixture (acetone, benzonitrile, benzene, toluene and naphthalene) much used by column manufacturers at that time. This test mixture conveniently evaluates stationary phase selectivity, efficiency and resolution. A second test mixture, containing an acidic compound (phenol) and two basic compounds (aniline and N,N-dimethylaniline) has also been used, which evaluates the silanol activity of the new stationary phases. More recently, Tanaka and co-workers, ${ }^{56}$ Engelhardt and Jungheim ${ }^{57}$ and NIST ${ }^{58}$ tests have also been used, to permit comparisons with data from the literature. ${ }^{59}$

The initial chromatographic results obtained with the first PMOS phases were very promising, taking into account, primarily, the low cost and simplicity of their preparation. In a subsequent study, the same authors evaluated the chemical stability of the PMOS phase through the monitoring of chromatographic parameters of some of these same compounds while passing a mobile phase of methanol:water (50:50, v/v) through the column. Chromatographic performance of the compounds showed little variation after passage of 5000 column volumes of mobile phase, indicating that the PMOS stationary phase was stable under neutral conditions. ${ }^{60}$

Collins et al.,$^{61}$ studying the coating of silica particles by PMOS, concluded that the polymer deposition on the silica pore surface initially occurs as "plugs" and is not a homogeneous and uniform layer, exposing some residual sites to interaction with sample components. One alternative to fix this problem was the use of an immobilization step to induce formation of a more uniform layer and to retain larger quantities of polymer on the silica

(b)

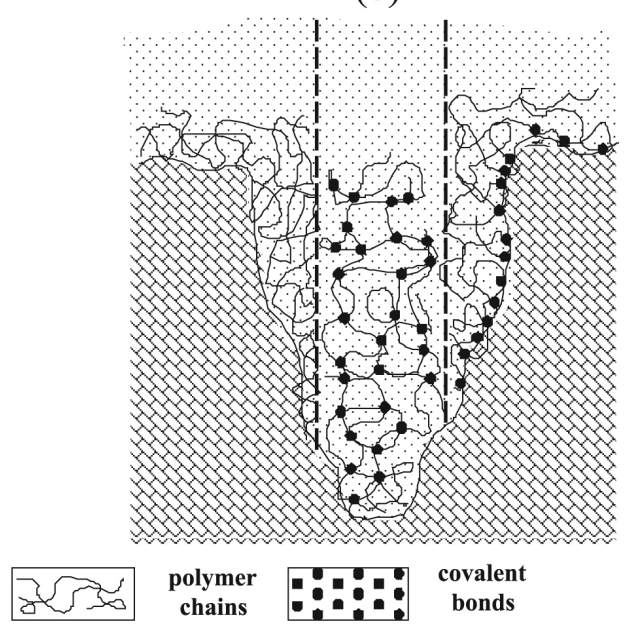

Figure 3. Types of polymeric deposition on a chromatographic support. (a) Coating of the pore walls or (b) filling of the pore volume. Adapted from Reference 53. 
surface, hindering the access of analytes to residual silanols and minimizing polymer loss with a continuous passage of the mobile phase. According to a study by Jardim et al. ${ }^{62}$ an immobilization step significantly contributes to obtaining uniform and thicker polymer layers on the silica surfaces, promoting better symmetry for the basic analyte peaks and maximizing the column efficiency in a chromatographic separation. Procedures that have been used by LabCrom for polymer immobilization onto silica support particles are: thermal treatment, ${ }^{63-66}$ gamma radiation ${ }^{55,62,67,68}$ and microwave irradiation. ${ }^{69-71}$

The quantities of polymer sorbed on the support surface are a predominant factor for obtaining stationary phases that provide efficient chromatographic separations. If, on the one hand, larger quantities of polymer provide a stationary phase with better protection of the residual groups against interactions with sample components, on the other hand, these larger quantities result in a thicker layer, blocking some support pores and making the mass transfer process slower. Therefore, the polymeric immobilization process should ensure a compromise between a thin film that does not block or restrict entry to the pores to maintain the overall structure and connectivity of the silica support, resulting in rapid mass transfer in the polymeric film; and a uniform polymeric layer to completely cover the non specific sites of the support, making it inaccessible to the analytes, as well as impermeable to basic mobile phases. Among the immobilization procedures studied in LabCrom, the use of thermal treatments has shown very good results, especially due to the ease of execution and the simple instrumentation required.

Tonhi et $a l .{ }^{72-74}$ prepared stationary phases using different immobilization procedures for attachment PMOS onto the silica support. Chromatographic results indicated that the PMOS phases obtained by thermal treatment and by gamma or microwave radiations did not show significant differences in their chromatographic performance, however, the thermally immobilized stationary phase showed higher chemical stability with neutral and slightly alkaline mobile phases, suggesting better uniformity of the polymeric layer. $^{74}$

The principle of thermal immobilization is based on heating of materials by conduction, in which a tube containing the recently prepared stationary phase is heated, with the heat being transferred to the materials inside. Thermal treatment accelerates the immobilization of polymers onto the silica surfaces, helping in the sorption process of more uniform polymeric layers. The thermal immobilization procedure was thoroughly investigated by Bottoli et al. ${ }^{63-66}$ using different immobilization temperatures and times to retain PMOS on different silica surfaces. These authors showed that stationary phases with maximum chromatographic performance can be prepared once there is an appropriate adjustment of the thermal immobilization conditions. ${ }^{64} \mathrm{~A}$ high immobilization temperature $\left(>150^{\circ} \mathrm{C}\right)$ significantly increases the quantity of polymer on the silica surfaces but reduces the chromatographic performance of the stationary phase. Recent studies have optimized the immobilization of PMOS onto the silica surfaces using a sequence of different heating steps in a single thermal immobilization procedure. ${ }^{75}$ These results have shown that, after a preliminary immobilization step at $100{ }^{\circ} \mathrm{C}$ for a controlled time, immobilization temperatures as high as $300{ }^{\circ} \mathrm{C}$ can be used, yielding stationary phases with substantial reduction of silanophilic activity while maintaining excellent chromatographic performances.

LabCrom has investigated several polymers other than PMOS, such as poly(methyloctadecylsiloxane) (PMODS): ${ }^{64,67}$ poly(dimethylsiloxane) (PDMS), ${ }^{60,76,77}$ poly(methylphenylsiloxane) (PMPS) ${ }^{78}$ poly(methyltetradecylsiloxane) (PMTDS) ${ }^{77}$ and polybutadiene (PBD), ${ }^{69,79,80}$ all immobilized onto silica particles for use as RP-HPLC stationary phases. These polymers have resulted on silicabased stationary phases with different selectivities due to the particular retention characteristics provided by each polymer for different analytes.

Chromatographic silica particles have also had significant changes during the 15 years of the stationary phase development by LabCrom, following the advances of commercial HPLC stationary phase technology. Irregular silica particles with $10 \mu \mathrm{m}$ diameter and high levels of metal impurities ("type A" silica), used the 1980's and early 1990's were gradually replaced by smaller particles $(8,7$ and $5 \mu \mathrm{m})$ with more regular (spherical) shapes and lower contents of metal impurities ("Type B" silica). These changes of particle properties have permitted a significant improvement in chromatographic performance of all HPLC stationary phases, mainly because decreasing particle size increases column efficiency and because the uniformity of spherical silica particles permits more uniform packing.

In spite of the advances in stationary phase preparation with the introduction of immobilization procedures, use of polymers with different selectivities and use of silica particles with greater homogeneity and lower metal impurity contents, the chemical stability of the immobilized polymer stationary phases still remains lower than that of some commercial stationary phases. To increase the chemical stability of the immobilized polymer stationary phases, LabCrom developed metalized silica supports. ${ }^{70,81-84}$ This material is based on deposition of metal oxide layers, such as zirconia and titania, onto presynthesized chromatographic silica particles. The principal characteristic of metalized silica is the combination of the 
appropriate properties of chromatographic silica, such as diameter, porosity and mechanical strength, with the higher chemical stability of metal oxides. ${ }^{85,86}$ The technology developed for the preparation of silica-based immobilized polymer stationary phases has been successfully transferred to the preparation of metalized silica-based phases.

\section{Metalized Silica-Based Immobilized Polymer Stationary Phases}

The initial studies with metalized silica supports were performed at LabCrom with the preparation of PMODS ${ }^{87}$ and PMOS $^{88}$ sorbed onto zirconized silica. The zirconized silica support was prepared by refluxing of zirconium(IV) tetrachloride in toluene with chromatographic silica. However, the quantity of zirconium attached to silica was extremely low, c.a. $1.5 \%$. A PMODS ${ }^{87}$ or $\mathrm{PMOS}^{88}$ layer was then sorbed onto the zirconized-silica support. The resulting stationary phases showed chromatographic performance similar to those obtained on bare silicabased stationary phases using the same polymers. These results indicated great promise for using this modified material as a chromatographic support. However, since the polymers were not immobilized, the retention parameters of compounds with these phases had low repeatability with continuous passage of mobile phase.

Jardim and co-workers ${ }^{81,82}$ replaced zirconium(IV) tetrachloride with zirconium(IV) tetrabutoxide for the preparation zirconized silica supports, increasing the quantity of zirconium attached to the silica to approximately $15 \%$. Moreover, these authors immobilized the PMOS onto the zirconized silica using gamma radiation to improve the repeatability of retention parameters for different compounds from analysis to analysis. Initial tests of chemical stability of these zirconized silica-based phases were very promising, resulting in significantly greater stability for zirconized-silica based stationary phase using alkaline mobile phases. ${ }^{82}$

Simultaneous with the development of sorbed and gamma immobilized PMOS stationary phases based on zirconized silica supports, Collins and co-workers ${ }^{83,84,89}$ prepared a new type of stationary phase based on the immobilization of PMOS onto titanized silica particles. The procedure for obtaining the titanized silica support was very similar to that used for the zirconized silica by Melo and Jardim. ${ }^{81}$ However, the quantity of titanium incorporated to silica was much lower, only $5 \%$. These authors also gamma immobilized a thin PMOS layer onto this support and the resulting stationary phase showed similar chromatographic performance to bare silica-based phases while their chemical stabilities were similar to the zirconized-silica based PMOS stationary phase using methanol:sodium hydroxide solutions at $25^{\circ} \mathrm{C} .{ }^{84}$

After these successes with metalized silica-based PMOS stationary phases, other polymers and immobilization procedures were used for preparation of new metalized silica-based stationary phases. Morais and $\mathrm{Jardim}^{70}$ studied the behavior of stationary phases prepared by immobilization of polybutadiene onto titanized silica particles using microwave irradiation. The titanized silica support was prepared by reaction of the chromatographic silica with titanium(IV) tetrabutoxide, resulting in $9 \%$ of titanium atoms attached to the silica. Fonseca et al..$^{90}$ used thermal treatments or microwave irradiation as procedures to immobilize PMOS onto titanized-silica particles (9\% titanium). The stationary phases also showed similar chromatographic performance to the bare silica-based PMOS stationary phases.

Fonseca et al. ${ }^{91}$ developed an accelerated stability test, using higher temperatures and mobile phases containing inorganic salts to evaluate the chemical stability of the stationary phases prepared at LabCrom. These conditions were used to accelerate the degradation of the stationary phase, reducing the evaluation time and, principally, the consumption of the solvents used in the stability tests. These more drastic conditions have permitted rapidly obtaining results of column lifetimes without loss of information, when compared to stability tests that use neutral mobile phases. ${ }^{91}$ This method has made it possible to quantify the improved stability of metalized silica-based stationary phases in relation to bare silica-based phases having the same immobilized polymer. These authors showed that a zirconized silica-based PMOS phase was stable to the passing of $600 \mathrm{~mL}$ of mobile phase at $\mathrm{pH} 10$, while the bare silica-based counterparts collapsed after $150 \mathrm{~mL}$. In a subsequent study, Faria et al. ${ }^{92}$ evaluated the chemical stability of the PMTDS stationary phases immobilized by different procedures onto titanized and zirconized silicas, using the accelerated stability test. The zirconized silicabased PMTDS phases, Si-Zr(PMTDS), were about 50\% more stable than titanized silica-based PMTDS phases, SiTi(PMTDS), and 400\% more stable than bare silica-based PMTDS phases, Si(PMTDS) (Table 1). When compared to the chemical stability of some commercial chemicallybonded phases, the zirconized-silica based PMTDS phases were more than competitive. ${ }^{92}$

Metalized silica supports provided a significant improvement in the chemical stability of immobilized polymer stationary phases, equating them to the stability of commercial stationary phases. ${ }^{109}$ However, the immobilization of polymer does not result in a uniform polymeric layer over the entire silica surface including in 
Table 1. Chromatographic stability of some LabCrom immobilizedpolymer and some commercial chemically bonded C8 or C18 stationary phases an alkaline mobile phase (pH 10)

\begin{tabular}{lccc}
\hline $\begin{array}{l}\text { Stationary } \\
\text { phases }\end{array}$ & $\begin{array}{c}\text { Column } \\
\text { volumes }\end{array}$ & $\begin{array}{c}\text { Mobile } \\
\text { phase / mL }\end{array}$ & Ref. \\
\hline Commercial "type A" 1* & 893 & 402 & 92 \\
Commercial "type A" 2* & 849 & 382 & 92 \\
Commercial "type B" 1* & 2036 & 916 & 92 \\
Commercial "type B" 2* & 1066 & 480 & 92 \\
Si(PMTDS) & 303 & 136 & 92 \\
Si-Ti(PMTDS) & 763 & 344 & 92 \\
Si-Zr(PMTDS) & 1248 & 562 & 92 \\
Si-Zr(PMTDS)ec & 2960 & 1332 & 97 \\
\hline
\end{tabular}

*Stationary phases listed according to the silica used as support. A: silica with higher metal impurity content; B: silica with lower metal impurity content.

all the pores. Some $\mathrm{OH}$ groups (from silica and/or from metal oxides) remain exposed. These residual groups retain strongly basic analytes, in some cases irreversibly, because these $\mathrm{OH}$ groups have their acidity increased due to the presence of metal (zirconium and titanium) atoms adjacent to the free silanol groups on the support. In addition, residual zirconol $(\mathrm{Zr}-\mathrm{OH})$ and titanol $(\mathrm{Ti}-\mathrm{OH})$ groups may behave as Lewis acid sites. ${ }^{81-94}$ Fonseca ${ }^{95}$ observed that separation of a test mixture containing phenol, N,N-dimethylaniline, naphthalene and acenaphthene with a titanized-silica based PMOS phase showed inversion of the elution order of N,N-dimethylaniline and naphthalene peaks due to the strong interaction between the basic analyte and residual groups. This interaction was more pronounced with lower immobilization times or temperatures of the thermal treatment used to prepare the PMOS phases. Figure 4 shows the chromatogram of a test mixture evaluated by Fonseca ${ }^{95}$ obtained with a stationary phase based on thermal immobilization of PMOS onto titanized silica, using two different immobilization times, 8 and $24 \mathrm{~h}$ at $120^{\circ} \mathrm{C}$. With the longer thermal immobilization time a greater amount of polymer is immobilized onto the silica particles, ensuring the normal elution order of the compounds evaluated.

Recently, new stationary phases have been developed at LabCrom that reduce residual acid groups from the support surface, such as immobilizing pre-synthesized polymers containing embedded polar groups in their structure onto zirconized silica particles. ${ }^{96}$ These stationary phases presented a balance between normal and reversed phase selectivities with significant reduction of residual groups. Another strategy that has been recently evaluated for reduction of the silanophilic activity of phases based on polymers immobilized onto metalized silica is to carry out an end-capping reaction after immobilization of the

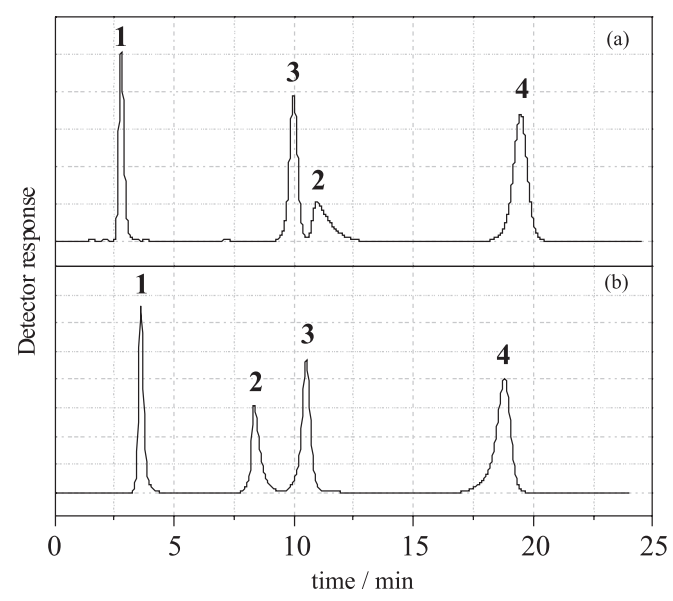

Figure 4. Chromatograms showing the separation of a test mixture with a thermally immobilized $\left(120^{\circ} \mathrm{C}\right)$ titanized-silica-based PMOS stationary phase using (a) $8 \mathrm{~h}$ and (b) $24 \mathrm{~h}$. Chromatographic conditions: mobile phase: methanol:water $(70: 30 \mathrm{v} / \mathrm{v})$, flow rate: $0.3 \mathrm{~mL} \mathrm{~min}^{-1}$; UV detection: $254 \mathrm{~nm}$; injection volume: $10 \mu \mathrm{L}$. Peak identifications: 1- phenol, 2- N,Ndimethylaniline, 3- naphthalene, 4-acenaphthene. Adapted from Ref. 95.

polymer. Faria et al..$^{97}$ prepared a stationary phase from the thermal immobilization of PMTDS onto zirconized silica particles and then submitted it to reaction with a mixture of trimethylchlorosilane and hexamethyldisilazane. This phase is denominated $\mathrm{Si}-\mathrm{Zr}(\mathrm{PMTDS})$ ec. Physicochemical and chromatographic characterizations showed that the residual activity of this stationary phase was significantly reduced due to the end-capping, providing symmetrical peaks with slightly basic analytes, such as N,Ndimethylaniline (Figure 5). In addition, the end-capped immobilized polymer stationary phase was approximately $150 \%$ more stable than its non end-capped counterpart and about $50 \%$ more resistant than some commercial chemically-bonded phases evaluated with the same alkaline mobile phases (Table 1).

\section{Stationary Phase Selectivity}

The first chemically-bonded stationary phases for RP-HPLC had octadecyl (C18) chains, probably due to availability and low cost of chloro- or alkoxyoctadecylsilanes, which were widely used as reagents for other purposes. Thus, it was logical that chromatographers used this reagent in the preparation of their stationary phases, resulting in the first commercial RP-HPLC columns. Another reason for the great popularity of $\mathrm{C} 18$ was the high reactivity of the appropriate chlorosilane with the silanol groups from the silica support. This fact was very important in early HPLC development because the silica support had low surface areas and needed large amounts of organic ligands for stronger retention of analytes. Another potential advantage is the length of the organic chain that more 


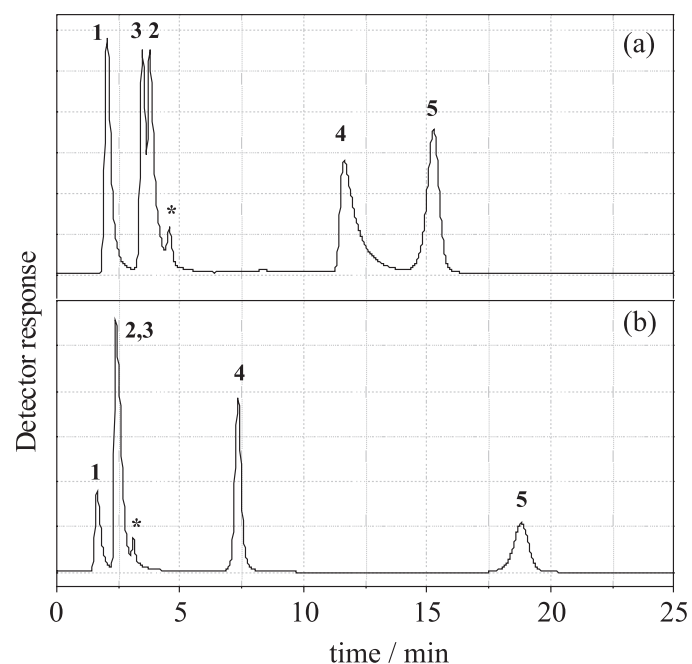

Figure 5. Chromatograms showing the separation of a test mixture with Si-Zr(PMTDS) phases: (a) not end-capped and (b) end-capped. Chromatographic conditions: mobile phase: methanol:water $(55: 45$ $\mathrm{v} / \mathrm{v}$ ), flow rate: $0.3 \mathrm{~mL} \mathrm{~min}^{-1}$; UV detection: $254 \mathrm{~nm}$; injection volume: $5 \mu$ L. Peak identifications: 1- uracil, 2- aniline, 3- phenol, 4- N,Ndimethylaniline and 5- naphthalene.

efficiently protects the silica support against acidic and basic mobile phases, when compared to the ligands with shorter chains. C18 stationary phase stability was very important because chromatographers could focus on the separation repeatability. ${ }^{8}$

With development of HPLC column technology, some drawbacks related to the $\mathrm{C} 18$ stationary phases became evident. Packing materials with high ligand density have shown stationary phase collapse when using highly aqueous mobile phases. In addition, stationary phases prepared with shorter chain organic ligands have provided better column efficiencies for polar and some non-polar compounds and reequilibrate more quickly after separations using gradient elution. These drawbacks of the octadecyl phase encouraged the study of new organic silanes for use in preparation of RP-HPLC stationary phases, as well as studies of the ideal length of the alkyl chain for chromatographic retention. Kováts and co-workers ${ }^{98}$ studied several alkylsilanes with different carbon chain lengths as stationary phases for HPLC, concluding that $\mathrm{C} 14$ groups result in ideal retention properties for chromatographic separations. According to these authors, ${ }^{98} \mathrm{C} 14$ stationary phases provide separations with higher column efficiencies and stabilities, optimal peak symmetries and better overall chromatographic retentions. However, although $\mathrm{C} 14$ or $\mathrm{C} 12$ groups have shown chromatographic properties more appropriate than C8 or C18 groups, chemically bonded C12 stationary phases have only recently appeared commercially.

The selectivity of immobilized polymer stationary phases is virtually identical to the selectivity of the chemically-bonded stationary phases containing similar functional groups. This occurs because most polymers used as stationary phases are polysiloxanes (Figure 6) that have high hydrocarbon compatibility. Thus, a polymer containing 18 carbon atoms on one side chain of each monomer (PMODS) appears equivalent to a $\mathrm{C} 18$ silane group during chromatographic separation. Moreover, polysiloxanes have high thermal stabilities, low vitreous transition temperatures and chemical and mechanical resistances appropriate to the chromatographic conditions in which the stationary phases are usually used. ${ }^{99}$

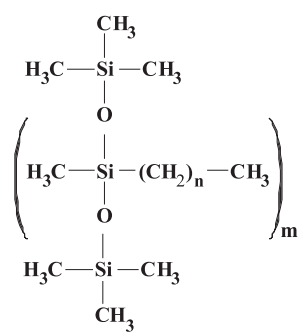

Figure 6. Chemical structure of polysiloxanes. $\mathrm{n}=$ number of carbon atoms present in side chain; 7- PMOS, 13- PMTDS, 17- PMODS, etc. $\mathrm{m}=$ number of monomer units.

LabCrom has been studying the chromatographic properties of $\mathrm{C} 14$ stationary phases, using poly(methyltetradecylsiloxane) (PMTDS). Tonhi et al. ${ }^{77}$ showed that the immobilization of PMTDS polymer onto a silica surface results in better chromatographic performance than PMOS or PMDS phases. Furthermore, immobilized PMTDS stationary phases showed better hydrophobic selectivity and lower silanophilic activity than the immobilized PMOS or PMDS stationary phases.

The selectivity of metalized silica-based immobilized polymer stationary phases is practically identical to their silica-based counterparts, as can be seen in Figure 7. Faria et al. ${ }^{93,94}$ prepared stationary phases by thermal and gamma immobilization of PMTDS onto metalized (zirconized and titanized) silica particles with similar hydrophobic selectivity for non-polar compounds to the immobilized-PMTDS silicabased phases. The chromatographic performance of the PMTDS metalized-silica based phases was also comparable to the silica-based phase, mainly in terms of column efficiencies, asymmetry factors and resolutions. The principal differences were observed in relation to chromatographic retention for basic analytes due to the presence of zirconium or titanium atoms in the silica support structure, increasing the acidity of residual $\mathrm{OH}$ groups (Figure 7). However, the tailing of peaks observed for basic compounds on the metalized silica-based phases can be easily minimized by using a phosphate-buffered mobile phase or a mobile phase containing a volatile amine as an additive. 


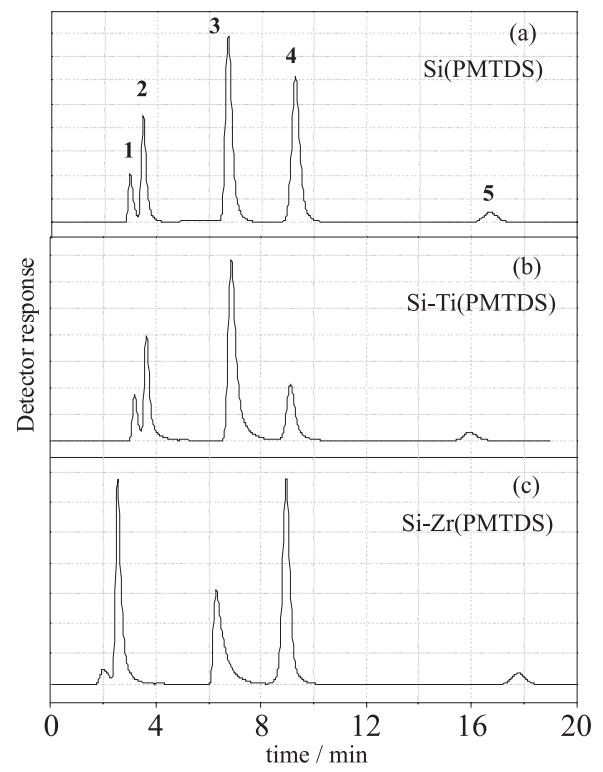

Figure 7. Chromatograms showing the separation of a test mixture with stationary phases prepared by thermal immobilization $\left(110^{\circ} \mathrm{C}\right)$ of PMTDS on (a) silica, (b) titanized silica and (c) zirconized silica particles. Chromatographic conditions: mobile phase: methanol:water (70:30 v/v), flow rate: $0.3 \mathrm{~mL} \mathrm{~min}^{-1}$; UV detection: $254 \mathrm{~nm}$; injection volume: $5 \mu \mathrm{L}$. Peak identifications: 1- uracil, 2- phenol, 3- N,N-dimethylaniline, 4naphthalene and 5- acenaphthene.

\section{Some Applications of the Immobilized Polymer Stationary Phases}

The applicability of the immobilized stationary phases has been evaluated recently for separations of compounds of environmental and human health interest, such as some classes of pesticides ${ }^{87,93}$ and pharmaceuticals..$^{94,100}$ These studies have been possible because of the high quality achieved by stationary phases developed by LabCrom. Pesticide and pharmaceutical compounds allow an appropriate evaluation of the viability of these stationary phases, because these compounds have large and often complex structures and include acidic, basic and neutral compounds with a wide range of polarities.

Pinto et al. ${ }^{87}$ used an immobilized PMODS zirconized silica-based stationary phase to separate seven herbicides with distinct characteristics; two acids (bentazon and fluazifop acid), four bases (cyanazine, simazine, atrazine and ametrin) and a non-polar compound (diuron). These authors reported symmetrical and resolved peaks for all herbicides using isocratic methanol:water mobile phase $(60: 40 \mathrm{v} / \mathrm{v})$ acidified with phosphoric acid at $\mathrm{pH}$ 4.6. Faria et al. ${ }^{93}$ prepared an immobilized-PMTDS zirconized-silica based stationary phase with potential for the separation of pesticides. Six pesticides, three herbicides and three fungicides commonly used on several different crops, were isocratically separated by a PMTDS phase using a methanol:water $(50: 50, \mathrm{v} / \mathrm{v})$ mobile phase. The total analysis time was less than 12 min (Figure 8).

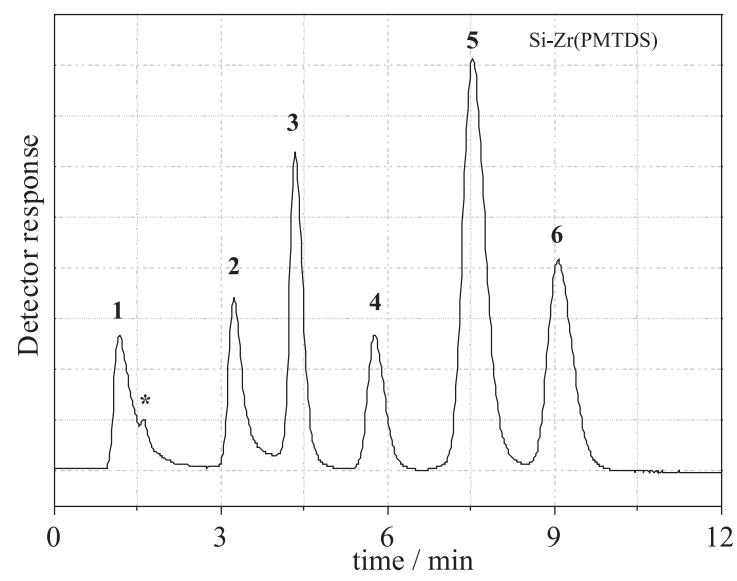

Figure 8. Chromatogram showing the separation of a pesticide mixture with a $\mathrm{Si}-\mathrm{Zr}(\mathrm{PMTDS})$ phase. Chromatographic conditions: mobile phase: methanol:water $(50: 50, \mathrm{v} / \mathrm{v})$, flow rate: $0.3 \mathrm{~mL} \mathrm{~min}^{-1}$; UV detection: $235 \mathrm{~nm}$; injection volume: $5 \mu \mathrm{L}$. Peak identifications: 1- imazethapyr, 2- cyanazine, 3- carboxin, 4- atrazine, 5- diuron, 6- linuron and *-subproduct from imazethapyr. Adapted from Ref. 93.

Immobilized-PMTDS zirconized-silica based stationary phases were also used to separate some pharmaceutical compounds. ${ }^{94,100}$ Pharmaceutical compounds normally present basic characteristics, allowing a more specific evaluation of the silanophilic activity presents on the immobilized polymer phase, principally with zirconized-silica based ${ }^{94}$ or titanizedsilica based ${ }^{100}$ stationary phases. The high acidity of the immobilized-polymer metalized-silica based stationary phases is responsible for the highly tailing peaks when eluting basic pharmaceuticals. On the other hand, the higher hydrolytic stability of metalized silica-based phases allows using more alkaline mobile phases, for example using mobile phase buffered at $\mathrm{pH}>8$, which is not recommended for silica-based stationary phases. Alkaline mobile phases buffered at $\mathrm{pH} 11$ and 12 are normally used for efficient separations of some basic pharmaceuticals with symmetrical peaks and good resolutions. These mobile phase conditions provide shorter analysis times and higher column efficiencies in the chromatographic separations, as can be seen in the chromatograms of Figure 9a and 9b.

Currently, some other applications using immobilizedpolymer stationary phases are under study, such as monitoring of multiresidue pesticides in some foods (strawberry, sugarcane, etc.) and the analysis of pharmaceuticals in human plasma. 


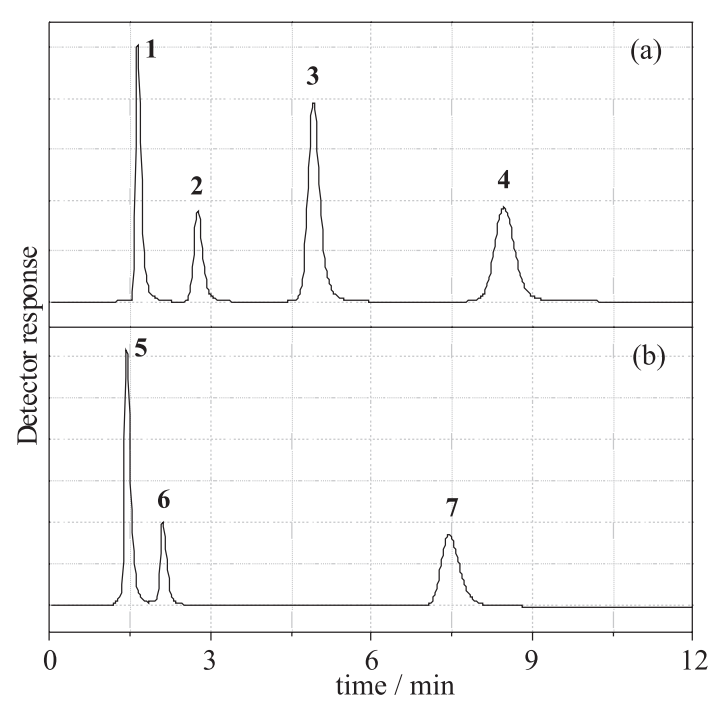

Figure 9. Chromatograms showing the separation of pharmaceutical compounds with (a) Si-Zr(PMTDS) and (b) Si-Ti(PMTDS) phases. Chromatographic conditions: mobile phases: (a) methanol:0.02 $\mathrm{mol} \mathrm{L}^{-1}$ $\mathrm{K}_{2} \mathrm{HPO}_{4} / \mathrm{KH}_{2} \mathrm{PO}_{4}, \mathrm{pH} 11(50: 50, \mathrm{v} / \mathrm{v})$, flow rate: $0.6 \mathrm{~mL} \mathrm{~min}{ }^{-1}$ and (b) methanol:0.02 $\mathrm{mol} \mathrm{L}^{-1} \mathrm{~K}_{2} \mathrm{HPO}_{4} / \mathrm{KH}_{2} \mathrm{PO}_{4}, \mathrm{pH} 12$ (70:30, v/v), flow rate: $0.2 \mathrm{~mL} \mathrm{~min}^{-1}$; UV detection: $254 \mathrm{~nm}$; injection volume: $5 \mu \mathrm{L}$. Peak identifications: 1- paracetamol, 2- ibuprofen, 3- lorazepan, 4- alprazolan, 5- cephalexin, 6- prilocaine and 7- amitriptyline. Adapted from Ref. 94 and Ref. 100, respectively.

\section{Immobilized-Polymer Sorbents for Solid Phase Extraction}

Sorbents for solid phase extraction (SPE) have been prepared at LabCrom using the same technology as used for preparation of RP-HPLC stationary phases, mixing a presynthesized polymer solution with larger silica particles, ${ }^{101-108}$ followed by an immobilization procedure. The silica particles used for preparation of SPE sorbents usually have diameters ranging from 35 to $75 \mu \mathrm{m}$, which allow faster eluent flows without excessive pressure, as is conventionally applied in SPE. The great attraction of these immobilized-polymer sorbents is their ease of preparation, as it is quite simple and does not require sophisticated equipment or materials. Furthermore, the preparation of these SPE sorbents uses low cost reagents (silica, polymers and solvents) and materials (syringes, frits, etc.), making these sorbents an alternative to the use of commercial sorbents. Moreover, the recovery results obtained for several analytes from different matrices as well as the concentration factors have been equivalent to those obtained with commercial SPE cartridges. Queiroz et al. ${ }^{101}$ prepared SPE cartridges by packing $500 \mathrm{mg}$ of gammaimmobilized PMODS sorbent into syringes ( $1 \mathrm{~mL}$ ), the sorbent being retained by two polyethylene frits. These SPE cartridges were used for offline extraction of six herbicides (benomil, tebuthiuron, simazine, atrazine, ametrin, and diuron) from drinking water samples. Recoveries ranged from 73 to $103 \%$ and repeatability levels had an RSD (relative standard deviation) less than $16 \%$. These authors compared the laboratory-made cartridges to Supelclean ${ }^{\circledR}$ cartridges (Supelco) for extraction of these six herbicides from drinking water, obtaining similar repeatability and recoveries for both the laboratory-made and the commercial cartridges.

The broad availability of pre-synthesized polymers has made possible the efficient fine-tuning of immobilized-polymer SPE sorbent selectivity for some applications. ${ }^{103,105-108}$ Melo et al. ${ }^{103,105}$ prepared SPE sorbents by immobilization of a polymer containing a mix of polar groups $\left(\mathrm{NH}_{2}\right)$ and hydrophobic groups (propylsiloxane) for extraction of pesticides from grape and tomatoes samples. Recovery results using these balanced-polarity cartridges were better than those obtained with commercial C18 or laboratory-made PMODS cartridges. Vigna et al. ${ }^{107}$ and Faria et al. ${ }^{108}$ prepared SPE sorbents by thermal immobilization of alkylsiloxanes with different carbon chain lengths, PMOS and PMTDS, respectively, for extraction and subsequent methodology validation of pesticides from water samples. In both cases, the immobilized polymer sorbents presented better recovery results with higher repeatability than obtained using commercial C18 SPE cartridges. Other applications of laboratory-made immobilized-polymer cartridges have also involved efficient extraction and concentration of several different compounds, such as lead ${ }^{104}$ from water and pesticides from water, ${ }^{101}$ urine ${ }^{102}$ and food samples (grapes, ${ }^{103}$ tomatoes ${ }^{105}$ ), indicating the universality of this method for both concentration and clean-up procedures.

\section{Conclusions and Perspectives}

The exponential development of high-performance liquid chromatography is closely related to the many advances in stationary phase technology. Stationary phases have significantly evolved in the last two decades, justifying the great popularity currently achieved by RP-HPLC. Different stationary phases were introduced in this period as described in this review. Immobilized-polymer stationary phases based on bare silica coupled to the use of efficient techniques for the immobilization of the polymer onto the silica surfaces as well as the advent and use of metalized silica supports have significantly extended the lifetime of stationary phases and are responsible for improvements in performance and stability of the immobilized-polymer phases. Furthermore, the easy and simple preparation of these immobilized polymer phases, using low cost materials and reagents, compared to those used in chemically-bonded stationary phases, together with the wide variety of polymers give these 
low cost packing materials a high potential to overcome some historical problems of RP-HPLC.

In spite of great advances obtained with immobilizedpolymer stationary phases, more applications involving complex situations still are needed. Furthermore, chemically stable stationary phases having less acidic residual groups might be obtained with the preparation of metalized silica supports using other metal oxides, such as hafnia, ceria, alumina or magnesia or using other functional polymers, containing different groups embedded onto the polymer structure, such as fluoroalkyl, alkylene oxide and mercaptoalkyl groups.

\section{Acknowledgments}

The authors acknowledge financial support and fellowships from the Fundação de Amparo à Pesquisa do Estado de São Paulo (FAPESP) and the Conselho de Desenvolvimento Científico e Tecnológico (CNPq) and also the postdoctoral, graduate, and undergraduate students whose work provided some of the data reported herein.

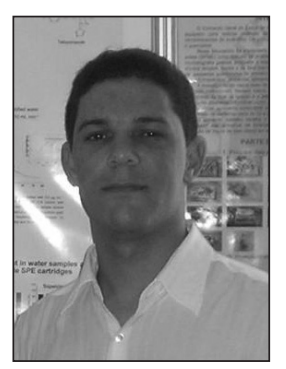

Anizio M. Faria was born in 1977 and got his bachelor's and licenciate degrees at the Federal University of Viçosa, Brazil, in 2001. Following a Masters's degree in agrochemistry at the Federal University of Viçosa, in 2003, he obtained a doctorate in Analytical Chemistry at the State University of Campinas (Unicamp), in 2006. He worked as a postdoctoral fellow in the group of Prof. Carol Collins and Prof. Isabel Jardim in the Research Laboratory for Liquid Chromatography at Unicamp, until 2008 when he assumed a position in the Faculty of Integrated Sciences at Pontal of the Federal University of Uberlândia, where he is currently associate professor and develops research in the field of chromatographic materials, more specifically development of new reversed stationary phases for liquid chromatography. He was a collaborative professor in the Department of Analytical Chemistry at Chemistry Institute of Unicamp between 2007-2009.

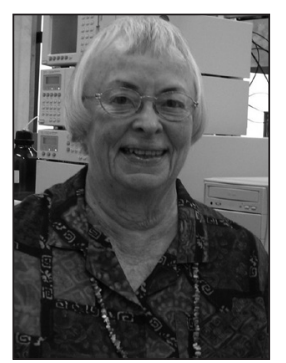

Carol Collins, born in the United States, obtained her B.S. from Bates College in 1952 and her Ph.D. in Physical and Organic Chemistry from Iowa State University in 1958. She did a post-doctorate at the University of Wisconsin (1958-1961) in these same areas and initiated studies in radiation chemistry and hot-atom chemistry at Brookhaven National Laboratory (USA) and at the Université de Louvain (Belgium). Subsequently she worked at several nuclear centers (State University of New York at Buffalo, USA, Taiwan, the Philippines and Indonesia), on projects of radiochemistry, using gas and liquid chromatographies. She joined the faculty of the Institute of Chemistry of the State University of Campinas in 1974 and became a Full Professor in 1988. She is currently an Invited Professor. Her present research activities principally involve high performance liquid chromatography, with emphasis on the preparation and evaluation of new stationary phases. She has published more than 160 papers in indexed journals as well as 14 book chapters, coordinated the publication of two books on chromatography and supervised more than 50 theses and dissertations. She received the first Marie Curie Fellowship from the American Association of University Women, is classified IA by CNPq and is a Titular member of the Brazilian Academy of Science.

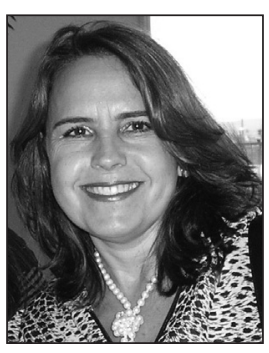

Isabel Cristina S. F. Jardim graduated in Chemistry in 1976 and obtained her doctorate in science in 1983, working with radiochemistry at the Institute of Chemistry of the State University of Campinas (Unicamp). Since 1981 she has been a professor of the Chemistry Institute of Unicamp and is now a Full Professor in the Analytical Chemistry Department. She teaches undergraduate and graduate classes for students in the Chemistry, Chemical Engineering and Food Engineering programs and also teaches extension courses and is a thesis supervisor in the graduate program in Analytical Chemistry. Her research interests are in separations using High Performance Liquid Chromatography, including stationary phase preparation, characterization and application as well as in the development and validation of methods for the determination of pharmaceuticals and pesticides in many types of matrices: water, soil, biological fluids and fruits. She has supervised master's dissertation and doctorate thesis and scientific initiation projects as well as several postdoctoral projects and has presented the results at national and international scientific congresses, as well as having published 68 papers on her work. She is the author of six book chapters and has solicited three patents.

\section{References}

1. Snyder, L. R.; J. Chromatogr. Sci. 1969, 7, 595.

2. Snyder, L. R.; Kirkland, J. J.; Glajch, J. L.; Practical HPLC Method Development, $2^{\text {nd }}$ ed., Wiley: New York, 1997. 
3. Halasz, I.; Sebestian, I.; Angew Chem., Int. Ed. 1969, 8, 453.

4. Locke, D. C.; Schmermund, J. T.; Banner, B.; Anal. Chem. 1972, 44, 90.

5. Kirkland, J. J.; DeStefano, J. J.; J. Chromatogr. Sci. 1970, 8, 309.

6. Kirkland, J. J.; J. Chromatogr. Sci. 1971, 9, 206.

7. Vansant, E. F.; van der Voort, D.; Vrancken, C.; Characterization and Chemical Modification of the Silica Surface, Elsevier: New York, 1995.

8. Kirkland, J. J.; J. Chromatogr., A 2004, 1060, 9.

9. Engelhardt, H.; Blay, Ch.; Saar, J.; Chromatographia 2005, 62, S19.

10. Majors, R. E.; LC-GC North Am. 2000, 18, 1214.

11. Smith, R. M.; J. Chromatogr. 1984, 291, 372.

12. Dawkins, J. V.; Lloyd, L. L.; Warner, F. P.; J. Chromatogr. 1986, 352, 157.

13. Viron, C.; André, P.; Dreux, M.; Lafosse, M.; Chromatographia 1999, 49, 137.

14. Mao, Y.; Fung, B. M.; J. Chromatogr., A 1997, 790, 9.

15. Pesek, J. J.; Matyska, M. T.; J. Chromatogr., A 2002, 952, 1.

16. Nawrocki, J.; Dunlap, C.; Li, J.; McNeff, C. V.; McCormick, A.; Carr, P. W.; J. Chromatogr., A 2004, 1028, 31.

17. Tani, K.; Suzuki, Y.; J. Chromatogr., A 1996, 722, 129.

18. Jiang, Z.; Zuo, Y.; Li, R.; Yu, J. C.; Chem. Anal. (Warsaw) 2004, 49, 551.

19. Li, J.; Carr, P. W.; Anal. Chem. 1996, 68, 2857.

20. Li, J.; Hue, Y.; Carr, P. W.; Anal. Chem. 1997, 69, 3884.

21. Zhao, J.; Carr, P. W.; Anal. Chem. 2000, 72, 302.

22. Dunlap, C. J.; Mcneff, C. V.; Stoll, D.; Carr, P. W.; Anal. Chem. 2001, 73, 598A.

23. Meyer, V. R.; Practical High-Performance Liquid Chromatography, $2^{\text {nd }}$ ed., Wiley: New York, 1996.

24. Nawrocki, J.; Dunlap, C.; McCormick, A.; Carr, P. W.; J. Chromatogr., A 2004, 1028, 1.

25. Nawrocki, J.; Rigney, M. P.; McCormick, A.; Carr, P. W.; J. Chromatogr., A 1993, 657229.

26. Neue, U. D.; Walter, T. H.; Alden, B. A.; Jiang, Z.; Fisk, R. P.; Cook, J. T.; Glose, K. H.; Carmody, J. L.; Grassi, J. M.; Cheng, Y. F.; Lu, Z.; Crowley, R.; Am. Lab. 1999, 31, 36.

27. Cheng, Y. F.; Walter, T. H.; Lu, Z.; Iraneta, P.; Alden, B. A.; Gendreau, C.; Neue, U. D.; Grassi, J. M.; Carmody, J. L.; O’Gara, J. R.; Fisk, R. P.; LC-GC North Am. 2000, 18, 1162.

28. Wyndhan, K. D.; O'Gara, J. E.; Walter, T. H.; Glose, K. H.; Lawrence, N. L.; Alden, B. A.; Izzo, G. S.; Hudalla, C. J.; Iraneta, P. C.; Anal. Chem. 2003, 75, 6781.

29. Sudo, Y.; J. Chromatogr., A 1996, 737, 139.

30. Kirkland, J. J.; Henderson, J. W.; DeStefano, J. J.; van Straten, M. A.; Claessens, H. A.; J. Chromatogr., A 1997, 762, 97.

31. Unger, K. K.; Becker, N.; Roumellotis, P.; J. Chromatogr. 1976, 125,115

32. Sander, L. C.; Wise, S. A.; Crit. Rev. Anal. Chem. 1987, 18, 299.
33. Wirth, M. J.; Fatunmbi, H. O.; Anal. Chem. 1992, 64, 2783.

34. Wirth, M. J.; Fatunmbi, H. O.; Anal. Chem. 1993, 65, 822.

35. Glajch, J. L.; Kirkland, J. J.; LC-GC North Am. 1990, 8, 140.

36. Kirkland, J. J.; van Straten, M. A.; Claessens, H. A.; J. Chromatogr., A 1998, 797, 111.

37. Kirkland, J. J.; Martosella, J. D.; Henderson, J. W.; Dilks Jr., C. H.; Adams Jr., J. B.; Am. Lab. 1999, 31, 22.

38. Kirkland, J. J.; Henderson, J. W.; Martosella, J. D.; Bidlingmeyer, B. A.; Vasta-Russel, J.; Adams Jr., J. B.; LC-GC 1999, 17, 634.

39. O'Gara, J. E.; Alden, B. A.; Walter, T. H.; Petersen, J. S.; Niederländer, C. L.; Neue, U. D.; Anal. Chem. 1995, 67, 3809.

40. Silva, C. R.; Collins, C. H.; Jardim, I. C. S. F.; Airoldi, C.; J. Chromatogr., A 2004, 1030, 157.

41. Silva, R. B.; Silva, C. R.; Airoldi, C.; Collins, C. H.; Jardim, I. C. S. F.; Microchem. J. 2006, 82, 150.

42. Akapo, S. O.; Matyska, M. T.; Pesek, J. J.; J. Chromatogr., A 1998, 773, 53.

43. O’Gara, J. E.; Walsh, D. P.; Phoebe Jr., C. H.; Alden, B. A.; Bouvier, E. S. P.; Iraneta, P. C.; Capparella, M.; Walter, T. H.; LC-GC North Am. 2001, 19, 632.

44. Majors, R. E.; LC-GC North Am. 1998, 16, 228.

45. Schomburg, G.; Köhler, J.; Figge, H.; Deege, A.; BienVogelsang, U.; Chromatographia 1984, 18, 265.

46. Bien-Vogelsang, U.; Deege, A.; Figge, H.; Köhler, J.; Schomburg, G.; Chromatographia 1984, 19, 170.

47. Schomburg, G.; TrAC, Trends Anal. Chem. 1991, 10, 163.

48. Claessens, H. A.; van Straten, M. A.; J. Chromatogr., A 2004, 1060, 23.

49. Ohmacht, R.; Kele, M.; Matus, Z; Chromatographia 1989, 28, 19.

50. Hanson, M.; Unger, K. K.; TrAC, Trends Anal. Chem. 1992, 11, 368.

51. Janus, L.; Carbonnier, B.; Deratani, A.; Bacquet, M.; Crini, G.; Laureyns, J.; Morcellet, M.; New J. Chem. 2003, 17, 307.

52. Carbonnier, B.; Janus, L.; Lekchiri, Y.; Morcellet, M.; J. Appl. Polym. Sci. 2004, 91, 1419.

53. Petro, M.; Berek, D.; Chromatographia 1993, 37, 549.

54. Anazawa, T. A.; Jardim, I. C. S. F.; J. Liq. Chromatogr. 1994, 17, 1265 .

55. Anazawa, T. A.; Jardim, I. C. S. F.; J. Liq. Chromatogr. Relat. Technol. 1998, 21, 645.

56. Kimata, K.; Iwaguchi, K.; Onishi, S.; Jinni, K.; Eksteen, R.; Hosoya, M.; Araki, M.; Tanaka, N.; J. Chromatogr. Sci. 1989, 27, 721.

57. Engelhardt, H.; Jungheim, M.; Chromatographia 1990, $29,59$.

58. Sander, L.; Wise, S. A.; J. Sep. Sci. 2003, 26, 283.

59. Euerby, M. E.; Peterson, P.; J. Chromatogr., A 2003, 994, 13.

60. Anazawa, T. A.; Carraro, F.; Collins, K. E.; Jardim, I. C. S. F.; J. Chromatogr., A 1995, 697, 159.

61. Collins, K. E.; Granja, M. L. M. M.; Pereira Filho, R. G.; Anazawa, T. A.; Jardim, I. C. S. F.; Chromatographia 1997, $45,99$. 
62. Jardim, I. C. S. F.; Collins, K. E.; Anazawa, T. A.; J. Chromatogr., A 1999, 849, 299.

63. Collins, K. E.; Sá, A. L. A.; Bottoli, C. B. G.; Collins, C. H.; Chromatographia 2001, 53, 661.

64. Bottoli, C. B. G.; Chaudhry, Z. F.; Fonseca, D. A.; Collins, K. E.; Collins, C. H.; J. Chromatogr., A 2002, 948, 121.

65. Bottoli, C. B. G.; Vigna, C. R. M.; Fischer, G.; Albert, K.; Collins, K. E.; Collins, C. H.; J. Chromatogr., A 2004, 1030, 217.

66. Bottoli, C. B. G.; Silva, C. R.; Collins, K. E.; Collins, C. H.; J. Liq. Chromatogr. Relat. Technol. 2005, 27, 407.

67. da Silva, M. C. H.; Jardim, I. C. S. F.; J. Liq. Chromatogr. Relat. Technol. 1998, 21, 2447.

68. Anazawa, T. A.; Collins, K. E.; Jardim, I. C. S. F.; J. Braz. Chem. Soc. 2004, 15, 116.

69. Lopes, N. P.; Collins, K. E.; Jardim, I. C. S. F.; J. Chromatogr., A 2004, 1030, 225.

70. Morais, L. S. R.; Jardim, I. C. S. F.; J. Chromatogr., A 2005, 1073, 127.

71. Kapor, M. A.; M.Sc. Dissertation, Universidade Estadual de Campinas, Brazil, 2001.

72. Tonhi, E.; Bachmann, S.; Albert, K.; Jardim, I. C. S. F.; Collins, K. E.; Collins, C. H.; J. Chromatogr., A 2002, 948, 97.

73. Tonhi, E.; Collins, K. E.; Collins, C. H.; J. Chromatogr., A 2002, 948, 109.

74. Tonhi, E.; Collins, K. E.; Collins, C. H.; J. Chromatogr, A 2003, 987, 93.

75. Vigna, C. R. M.; Bottoli, C. B. G.; Collins, K. E.; Collins, C. H.; J. Chromatogr., A 2007, 1156, 60.

76. Tonhi, E.; Collins, K. E.; Collins, C. H.; J. Chromatogr., A 2005, 1075, 87.

77. Tonhi, E.; Collins, K. E.; Collins, C. H.; J. Chromatogr., A 2006, $1119,135$.

78. Lourenço, J.; M.Sc. Dissertation, Universidade Estadual de Campinas, Brazil, 2005.

79. Lopes, N. P.; Collins, K. E.; Jardim, I. C. S. F.; J. Chromatogr., A 2003, 987, 77.

80. Lopes, N. P.; Collins, K. E.; Jardim, I. C. S. F.; J. Chromatogr., A 2004, 1030, 231.

81. Melo, L. F. C.; Jardim, I. C. S. F.; J. Chromatogr., A 1999, 845, 423.

82. Melo, L. F. C.; Collins, C. H.; Collins, K. E.; Jardim, I. C. S. F.; J. Chromatogr., A 2000, 869, 129.

83. Silva, R. B.; Collins, C. H.; J. Chromatogr., A 1999, 845, 417.

84. Silva, R. B.; Collins, K. E.; Collins, C. H.; J. Chromatogr., A 2000, 869, 137.

85. Faria, A. M.; Magalhães, D. R.; Collins, C. H.; Revista Matéria 2004, 9, 344.

86. Faria, A. M.; Magalhães, D. R.; Collins, K.E.; Collins, C. H.; Anal. Chim. Acta 2005, 550, 137.

87. Pinto, G. M. F.; Collins, K. E.; Jardim, I. C. S. F.; J. Liq. Chromatogr. Relat. Technol. 2002, 25, 205.
88. Zuin, C. F.; M.Sc. Dissertation, Universidade Estadual de Campinas, Brazil, 1998.

89. Silva, R. B.; Gushikem, Y.; Collins, C. H.; J. Sep. Sci. 2001, 24, 49.

90. Fonseca, D. A.; Collins, K. E.; Collins, C. H.; J. Chromatogr., A 2004, 1030, 209.

91. Fonseca, D. A.; Gutiérrez, H. R.; Collins, K. E.; Collins, C. H.; J. Chromatogr., A 2004, 1030, 149.

92. Faria, A. M.; Tonhi, E.; Collins, K. E.; Collins, C. H.; J. Sep. Sci. 2007, 30, 1844.

93. Faria, A. M.; Collins, K. E.; Collins, C. H.; J. Chromatogr., A 2006, 1122, 114.

94. Faria, A. M.; Collins, K. E.; Collins, C. H.; J. Chromatogr., A 2007, 1156, 51.

95. Fonseca, D. A.; Ph.D. Thesis, Universidade Estadual de Campinas, Brasil, 2004.

96. Magalhães, D. R.; Ph.D. Thesis, Universidade Estadual de Campinas, Brasil, 2005.

97. Faria, A. M.; Silva, C. R.; Collins, C. H.; Jardim, I. C. S. F.; J. Sep. Sci. 2008, 31, 953.

98. Szabó, K.; Ha, N. L.; Schneider, Ph.; Zeltner, P.; Kóvats, E. Sz.; Helv. Chim. Acta 1984, 67, 2128.

99. http//www.unitedchem.com/product.aspx $? \mathrm{P}=229$, accessed in May, 2006.

100. Faria, A. M.; Collins, K. E.; Collins, C. H.; Chromatographia 2008 67, 357.

101. Queiroz, S. C.N.; Melo, L. F. C.; Jardim, I. C. S. F.; J. Chromatogr., A 2002, 948, 171.

102. Pozzebon, J. M.; Queiroz, S. C. N.; Melo, L. F. C.; Kapor, M. A.; Jardim, I. C. S. F.; J. Chromatogr., A 2003, 987, 381.

103. Melo, L. F. C.; Collins, C. H.; Jardim, I. C. S. F.; J. Chromatogr., A 2004, 1032, 51.

104. Maltez, H. F.; Melo, L. F. C.; Queiroz, S. C. N.; Jardim, I. C. S. F. J.; Curtius, A. J.; Carasek, E.; Microchim. Acta 2004, 144, 17.

105. Melo, L. F. C.; Collins, C. H.; Jardim, I. C. S. F.; J. Chromatogr., A 2005, 1073, 75.

106. Queiroz, S. C. N.; Melo, L. F. C.; Jardim, I. C. S. F.; Quim. Nova 2006, 29, 637.

107. Vigna, C. R. M.; Morais, L. S. R.; Collins, C. H.; Jardim, I. C. S. F; J. Chromatogr., A 2006, 1114, 211.

108. Faria, A. M.; Maldaner, L.; Santana, C. C.; Jardim, I. C. S. F.; Collins, C. H.; Anal. Chim. Acta 2007, 582, 34.

109. Collins, C.H.; Silva, C.R.; Faria, A.M.; Collins, K.E.; Jardim, I.C.S.F.; J. Braz. Chem. Soc. 2009, 20, 604.

Received: April 30, 2008

Web Release Date: August 31, 2009

FAPESP helped in meeting the publication costs of this article. 